\title{
Estimation des contraintes dans les roches à partir de l'écaillage autour de trous de forage
}

L. LI

M. AUBERTIN

Département des Génies civil géologique et des mines Ecole polytechnique de Montréal

Case postale 6079 Succursale Centre-Ville Montréal, QC, H3C $3 A 7$
L'écaillage autour des trous de forage dans la roche a souvent été utilisé comme indicateur pour évaluer l'orientation et la grandeur de la contrainte principale majeure. Des études antérieures ont ainsi montré que la contrainte horizontale principale maximale

(perpendiculaire au trou) peut être estimée avec un critère de rupture bi-dimensionnel, comme le critère de Coulomb, lorsque les contraintes verticale et horizontale minimale sont déjà connues. Dans cet article, les auteurs présentent une nouvelle méthode pour estimer l'ampleur des contraintes horizontales maximale et minimale à partir de la géométrie de la zone de rupture autour d'un trou de forage, en utilisant un critère de rupture multiaxial appelé MSDP . Après avoir introduit la formulation du critère, la procédure proposée est décrite et appliquée à un cas schématisé. Puis la méthode est utilisée pour estimer la contrainte horizontale maximale, et validée à l'aide de résultats expérimentaux tirés de la littérature. Un des intérêts de cette méthode est qu'elle peut être utilisée aussi bien pour estimer les contraintes naturelles dans les massifs rocheux que les contraintes induites autour des excavations.

Mots clés : roche, critère de rupture, écaillage en trou de forage, contraintes naturelles, contraintes induites.

\section{Evaluation of in situ stresses from borehole breakouts in rocks}

Borehole breakouts have often been used as indicators to evaluate both the orientation and magnitude of the major principal stress. Past investigations have shown that the maximum horizontal (principal) stress around a vertical hole can be estimated using a $2 D$ failure criterion, such as the Coulomb criterion, if the vertical and minimum horizontal stresses are already known. In this paper, the authors present a new method to estimate the magnitude of both the maximum and minimum horizontal principal stresses from the geometry of a borehole breakout using a multiaxial failure criterion, named MSDP After recalling the criterion formulation, the proposed procedure is described and applied with an illustrative example. Then the method is used for the evaluation of the maximum horizontal principal stress and validated with laboratory test results taken from literature. The method can be used for the evaluation of natural stresses in rock mass as well as for induced stresses around openings.

Key words : rock, failure criterion, borehole breakout, in situ stress, induced stress. 


\section{LISTE DES SYMBOLES}

\begin{tabular}{|c|c|}
\hline$A_{1}, \mathrm{a}_{2}, \mathrm{D}$ & tère MSDP $_{4}$. \\
\hline $\mathrm{C}_{0}$ & résistance en compression uniaxiale. \\
\hline$d_{b}$ & : profondeur de l'écaillage de forage. \\
\hline E & : module de Young. \\
\hline $\mathrm{F}_{0}$ & $\begin{array}{l}\text { fonction définissant la forme de la surface } \\
\text { de MSDP dans le plan } \sqrt{J_{2}}-I_{1} \text {. }\end{array}$ \\
\hline$F_{n}$ & $\begin{array}{l}\text { fonction contrôlant la forme de la surface } \\
\text { de MSDP dans le plan } \pi \text {. }\end{array}$ \\
\hline$I_{1}$ & $\begin{array}{l}\text { premier invariant du tenseur de contrainte } \\
\sigma_{i j}\end{array}$ \\
\hline $\mathrm{J}_{2}$ & $\begin{array}{l}\text { deuxième invariant du tenseur de contrainte } \\
\text { déviatrice } S_{\text {ij. }}\end{array}$ \\
\hline$S_{i j}$ & : tenseur de contrainte déviatrice. \\
\hline $\mathrm{T}_{0}$ & $\begin{array}{l}\text { résistance de traction uniaxiale (valeur } \\
\text { négative). }\end{array}$ \\
\hline$\alpha$ & $\begin{array}{l}\text { pente de la portion linéaire du critère } \\
\text { MSDP dans le plan } \sqrt{J_{2}}-I_{1} \text {. }\end{array}$ \\
\hline$\phi_{D,}, \phi_{i}$ & $\begin{array}{l}\text { angles de frottement basique et résiduel } \\
\text { respectivement. }\end{array}$ \\
\hline$v$ & : coefficient de Poisson. \\
\hline$\theta$ & : angle de Lode. \\
\hline$\sigma_{1}, \sigma_{2}, \sigma_{3}$ & $\begin{array}{l}\text { : contraintes principales majeure, intermé- } \\
\text { diaire et mineure respectivement. }\end{array}$ \\
\hline$\sigma_{c}$ & $\begin{array}{l}\text { paramètre de la résistance en compression } \\
\text { uniaxiale utilisé }\left(\sigma_{c}=C_{0}\right) \text {. }\end{array}$ \\
\hline$\sigma_{h}, \sigma_{H}$ & $\begin{array}{l}\text { : contraintes principales horizontales } \\
\text { mineure et majeure respectivement. }\end{array}$ \\
\hline$\sigma_{i j}$ & : tenseur de contrainte. \\
\hline$\sigma_{t}$ & $\begin{array}{l}\text { paramètre de la résistance en traction } \\
\text { uniaxiale utilisé }\left(\sigma_{1}=\left|T_{0}\right|\right) \text {. }\end{array}$ \\
\hline$\sigma_{v}$ & : contrainte verticale. \\
\hline$\Theta_{b}$ & : angle de l'écaillage de forage. \\
\hline$\Theta$ & $\begin{array}{l}\text { angle entre le point } \mathrm{B} \text { et la direction de la } \\
\text { contrainte horizontale maximum } \sigma_{\mathrm{H}}\end{array}$ \\
\hline
\end{tabular}

1

\section{Introduction}

On observe souvent un certain écaillage autour des trous de forage dû à l'instabilité de la roche. Ceci induit usuellement des zones de rupture avec une géométrie symétrique des deux côtés du forage (Bell et Gough, 1979). Ce phénomène a été identifié il y a déjà plusieurs dizaines d'années (Leeman, 1964). Depuis lors, de nombreux auteurs ont montré que l'écaillage autour d'un trou de forage peut constituer un indicateur de la direction de la contrainte principale maximale dans le plan perpendiculaire à l'axe du trou (Bell et Gough, 1979). Plusieurs investigations ont été entreprises par divers auteurs, dont celles de Zoback et de ses collaborateurs (e.g. Anderson et al., 1985 ; Barton et al., 1985 ; Hickman et al., 1985 ; Zoback et al., 1985 ; Vernik et Zoback, 1992 ; Peska et Zoback, 1995) qui ont grandement contribué à établir une méthode afin d'évaluer la direction et l'ampleur de la contrainte principale maximale à partir des zones d'écaillage observées autour des trous.
Par rapport aux méthodes traditionnelles de mesure des contraintes, telles que la méthode de fracturation hydraulique ou les méthodes de surcarottage, la méthode basée sur l'écaillage autour de trous de forage est surtout avantageuse d'un point de vue économique. Il n'est donc pas surprenant que le phénomène ait attiré l'attention de nombreux chercheurs au fil des ans (e.g. Plumb et Hickman, 1985; Zheng et Cook, 1985 ; Maury, 1987 ; Mastin, 1988 ; Guenot, 1989 ; Plumb, 1989; Zheng et al., 1989; Cheatham, 1993; Haimson et Song, 1993 ; Lee et Haimson, 1993 ; Martin, 1993 ; Elata, 1997; Aglawe, 1999). En fait, les analyses sur l'écaillage autour des forages représenteraient plus de $25 \%$ des données utilisées dans l'élaboration de la carte mondiale sur les contraintes naturelles (Zoback, 1992). L'applicabilité de telles observations pour la détermination des contraintes a été confirmée par les résultats récents publiés par Song et Haimson (1997).

La plupart des investigations mentionnées ci-dessus sont basées sur des analyses bi-dimensionnelles avec des critères de rupture à deux contraintes principales, telles que l'expression de Coulomb par exemple. Or, Song et Haimson (1997) ont montré que l'influence de la contrainte principale intermédiaire n'est pas nécessairement négligeable, ce qui limite la signification de certaines analyses antérieures. Théoriquement, un critère 2D n'est valable que pour des conditions identiques à celles utilisées pour déterminer les propriétés de matériau, soit en général : $\sigma_{1}>\sigma_{2}=\sigma_{3}$ qui correspond à des essais CTC (compression triaxiale conventionnelle), avec $\sigma_{2}, \sigma_{2}$ et $\sigma_{3}$ les contraintes principales maximale, intermediaire et minimale respectivement. Cette condition est rarement rencontrée autour des ouvertures en massif rocheux. Hoskins (1969) a d'ailleurs démontré que l'extension directe des modèles bi-dimensionnels aux conditions triaxiales ne peut pas toujours être justifiée. Pour ces raisons, il s'avère nécessaire d'utiliser un critère multiaxial qui tienne compte de la nature tridimensionnelle de l'état des contraintes induisant la rupture afin d'évaluer les contraintes en place de façon plus réaliste (Li et al., 1999).

D'autre part, on a constaté que certaines caractéristiques des zones d'écaillage autour de trous de forage n'ont pas été totalement exploitées pour la détermination des contraintes naturelles. Par exemple, la plupart des investigations ne permettent d'estimer la contrainte principale horizontale maximale $\left(\sigma_{H}\right)$ autour d'un trou vertical que si l'on connait la contrainte dans l'axe du trou $\sigma_{v}$ (contrainte principale estimée à partir du poids de terre) et la contrainte principale horizontale minimale $\sigma_{h}$ (estimée à partir de mesures de fracturation hydraulique, par exemple). Haimson et Herrick (1985) ont toutefois proposé une méthode pour évaluer, à partir d'un calcul inverse, les contraintes horizontales maximale et minimale à partir des résultats expérimentaux du laboratoire, en adoptant une approche similaire à celle de Zoback et al. (1985). Leur approche est donc de nature bi-dimensionnelle. De plus, elle ne prend pas en compte la progression de la zone de fracturation, même si cet aspect est important (Mastin, 1984). Plus récemment, Qian et Pedersen (1991) ont proposé une méthode d'inversion pour estimer les rapports $\sigma_{\mathrm{h}} / \sigma_{\mathrm{y}}$ et $\sigma_{H} / \sigma_{v}$ à partir des directions des zones d'écaillage lorsque les forages ne sont pas parfaitement verticaux. Cette méthode fournit des résultats intéressants lorsque les zones d'écaillage observées ont des directions différentes le long d'un forage à inclinaison variable avec la profondeur. Cette méthode peut cepen- 
dant induire des erreurs importantes, notamment lorsque le champ de contraintes naturelles varie avec la profondeur (e.g. Yin et Cornet, 1994). Une méthode alternative est proposée dans ce qui suit.

Dans cet article, les auteurs rappellent d'abord la formulation d'un critère de rupture multiaxial, appelé $\mathrm{MSDP}_{\mathrm{u}}$, qui constitue une version mathématique modifiée du critère MSDP récemment proposé par Aubertin et Simon $(1996,1998)$. Une méthode pour évaluer la grandeur et la direction des contraintes principales maximale et minimale $\left(\sigma_{\mathrm{H}}\right.$ et $\left.\sigma_{\mathrm{h}}\right)$ est ensuite présentée et appliquée à partir d'un exemple illustratif simplifié en utilisant le critère de rupture précité. La méthode proposée est basée sur l'analyse de la stabilité d'un trou de forage vertical en adoptant les hypothèses suivantes:

- la contrainte verticale $\sigma$, est une contrainte verticale principale, parallèle à l'axe du trou de forage. Elle est évaluée à partir de mesures directes ou calculée selon les poids de terre ;

- la roche est élastique linaire, homogène et isotrope ; - les effets du temps et de l'environnement sont négligeables.

Comme cas particulier, on montre l'application de la méthode pour l'évaluation de la contrainte principale maximale avec des résultats d'essai de laboratoire tirés de la littérature.

\section{Le critère de rupture MSDP}

Un critère de rupture multiaxial, nommé MSDP (pour Mises-Schleicher \& Drucker-Prager), a récemment été développé (Aubertin et Simon, 1996, 1998). Un des avantages de ce critère, c'est que les quatre paramètres requis peuvent être obtenus à partir d'essais simples et indépendants : essais en compression uniaxial, essais de traction uniaxiale (ou équivalent), et essais d'inclinomètre ou de cisaillement sur des surfaces planes ; on peut aussi utiliser les résultats d'essais en compression biaxiale, en compression triaxiale conventionnelle, et en extension triaxiale pour obtenir et/ou confirmer les valeurs des paramètres de résistance de la roche.

Le critère MSDP a été développé de façon à représenter les principales caractéristiques de la rupture des roches en compression et en traction pour divers cheminements de contraintes. Ses attributs particuliers ont déjà été démontrés par Aubertin et Simon $(1996,1998)$; la bonne correspondance entre le critère et divers résultats expérimentaux a aussi été illustrée dans ces publications. Le critère MSDP, malgré sa simplicité relative et sa signification physique, comporte toutefois quelques limitations dues principalement à sa formulation mathématique (et non à ses capacités descriptives). Ces aspects ont été récemment revus par Aubertin et al. (1999a) et une formulation mise à jour, appelé MSDP (pour formulation MSDP unifiée), a été proposée. On peut exprimer cette version du critère comme suit :

$$
\sqrt{\mathrm{J}_{2}}-\mathrm{F}_{0} \mathrm{~F}_{\mathrm{n}}=0
$$

ou en forme explicite pour les roches peu poreuses:

$$
\sqrt{J_{2}}-b\left\{\frac{\alpha^{2}\left(I_{1}^{2}-2 a_{1} I_{1}\right)+a_{2}^{2}}{b^{2}+\left(1-b^{2}\right) \sin ^{2}\left(45^{\circ}-1.5 \theta\right)}\right\}^{1 / 2}=0
$$

où $\mathrm{F}_{0}$ donne la forme de la surface de rupture dans le plan $\sqrt{J_{2}}-I_{1}$, et $F_{n}$ est la fonction dans le plan octahédral $(\pi)$ perpendiculaire à l'axe hydrostatique. Dans ces équations, les paramètres de résistance du matériau sont définis par les équations suivantes:

$$
\begin{gathered}
\alpha=\frac{2 \sin \phi}{\sqrt{3}(3-\sin \phi)} \\
a_{1}=\left(\frac{\sigma_{c}-\sigma_{t}}{2}\right)-\left(\frac{\sigma_{c}^{2}-\left(\sigma_{t} / b\right)^{2}}{6 \alpha^{2}\left(\sigma_{c}+\sigma_{t}\right)}\right) \\
a_{2}=\left\{\left(\frac{\sigma_{c}+\sigma_{t} / b^{2}}{3\left(\sigma_{c}+\sigma_{t}\right)}-\alpha^{2}\right) \sigma_{c} \sigma_{t}\right]^{1 / 2}
\end{gathered}
$$

où $\sigma_{c}\left(=C_{p}\right)$ et $\sigma_{i}\left(=\mid T_{D}\right)$ ) sont les résistances uniaxiales en compression et en traction respectivement; la valeur de l'angle $\phi$ dans l'équation 2 est égale à $\phi$, ou $\phi$. (l'angle de frottement résiduel ou de base); le paramètre b est principalement relié à la forme de la surface de rupture dans le plan $\pi$ (souvent $b \cong 0,75$ ) ; $I$, est le premier invariant du tenseur des contraintes $\left(I_{4}=\right.$ $\left.\operatorname{tr}\left(\sigma_{1}\right)\right) ; J_{2}$ est le deuxième invariant du tenseur de contrainte déviatorique $\left(\mathrm{J}_{2}=\left(1 / 2 \mathrm{~S}_{1} \mathrm{~S}\right)\right) ; \theta$ est l'angle de Lode définit dans le plan octahédral $(\pi)$;

$\theta=\frac{1}{3} \sin ^{-1} \frac{3 \sqrt{3} J_{3}}{2 \sqrt{J_{2}^{3}}}=\tan ^{-1} \frac{\sigma_{1}+\sigma_{3}-2 \sigma_{2}}{\sqrt{3}\left(\sigma_{1}-\sigma_{3}\right)},\left(-30^{\circ} \leq \theta \leq 30^{\circ}\right)(5)$

La figure 1 montre schématiquement les composantes $F_{0}$ et $F_{\pi}$ du critère MSDP dans les plans $\sqrt{J_{2}}-I_{4}$ et $\pi$ respectivement; la figure $1 \mathrm{c}$ montre le critere dans le plan des contraintes biaxiales conventionnelles.

La valídité du critère MSDP a été récemment évaluée pour différents types de roches (Aubertin et al. 1999a). A titre d'exemples, la figure 2 montre le critère appliqué aux résultats obtenus sur un granite soumis à des essais de compression triaxiale conventionnelle, et la figure 3 montre la comparaison entre le critère et des résultats expérimentaux obtenus sur des cylindres creux de calcaire. On constate que le critère décrit bien la rupture des roches dans un espace multiaxial.

\section{3}

\section{Évaluation des contraintes à partir de l'écaillage}

Comme mentionné précédemment, les zones d'écaillage autour des trous de forage ont été fréquemment utilisées pour évaluer une des composantes du champ des contraintes naturelles. La plupart des investigations antérieures reposent sur l'utilisation du critère de Coulomb ou d'autres critères bi-dimensionnels simplifiés, qui ne sont pas nécessairement représentatifs pour de telles applications (e.g. Maury, 1987; Song et Haimson, 1997). Le critère MSDP ${ }_{u}$ présenté ci-dessus est pour sa part bien adapté pour évaluer la stabilité des ouvertures souterraines, incluant les trous de forage. 

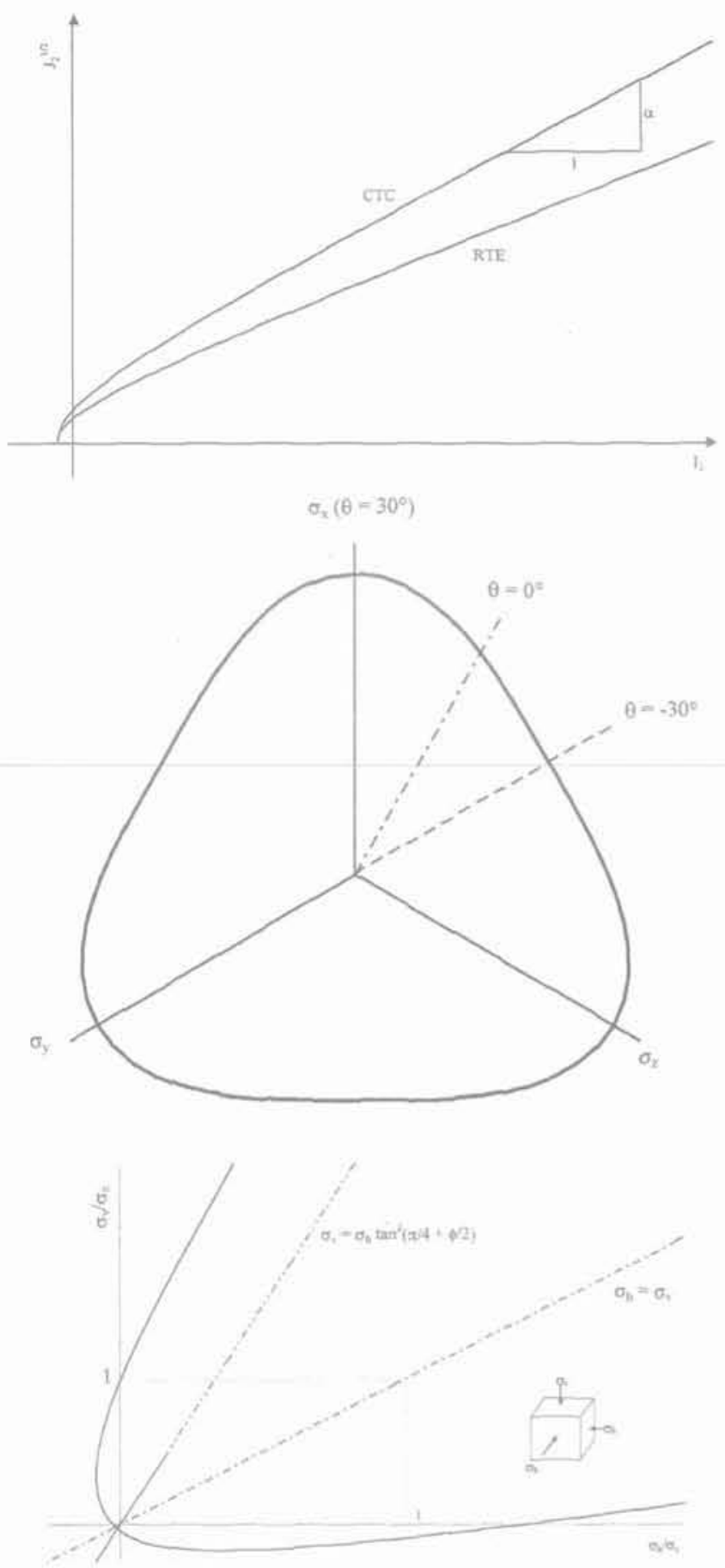

FG. Représentation schématique du critère MSDP $_{u}$ : (a) dans le plan $\sqrt{J_{2}}-I_{1}$; CTC :

compression triaxiale conventionnelle $(\theta=$ $\left.30^{\circ}\right)$; RTE : extension triaxiale $\left(\theta=-30^{\circ}\right)$; (b) dans le plan $\pi$; (c) dans le plan usuel des contraintes biaxiales.

Schematic representation of the MSDP

criterion: (a) in the $\sqrt{J_{2}}-I_{1}$ plane: CTC: conventional triaxial compression $\left(\theta=30^{\circ}\right)$ : RTE: reduced triaxial extension $\left(\theta=-30^{\circ}\right)$; (b) in the $\pi$ plane ; $(c)$ in the usual biaxial stress plane.

Les auteurs présentent ici une méthode utilisant à la fois l'angle et la profondeur de la zone d'écaillage autour d'un forage pour estimer les contraintes principales horizontales maximale $\sigma_{\mathrm{H}}$ et minimale $\sigma_{\mathrm{h}}$, en appliquant ce critère multiaxial. La contrainte verticale

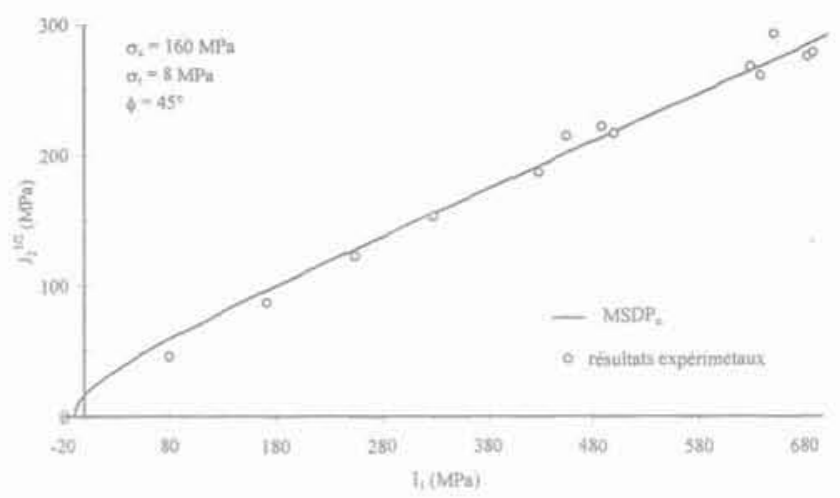

FG.2 Le critère MSDP appliqué à la rupture d'un granite sous "CTC (données tirées de Schwartz, 1964).

The MSDP criterion applied to granite submitted to CTC loadings (data taken from Schwartz, 1964)
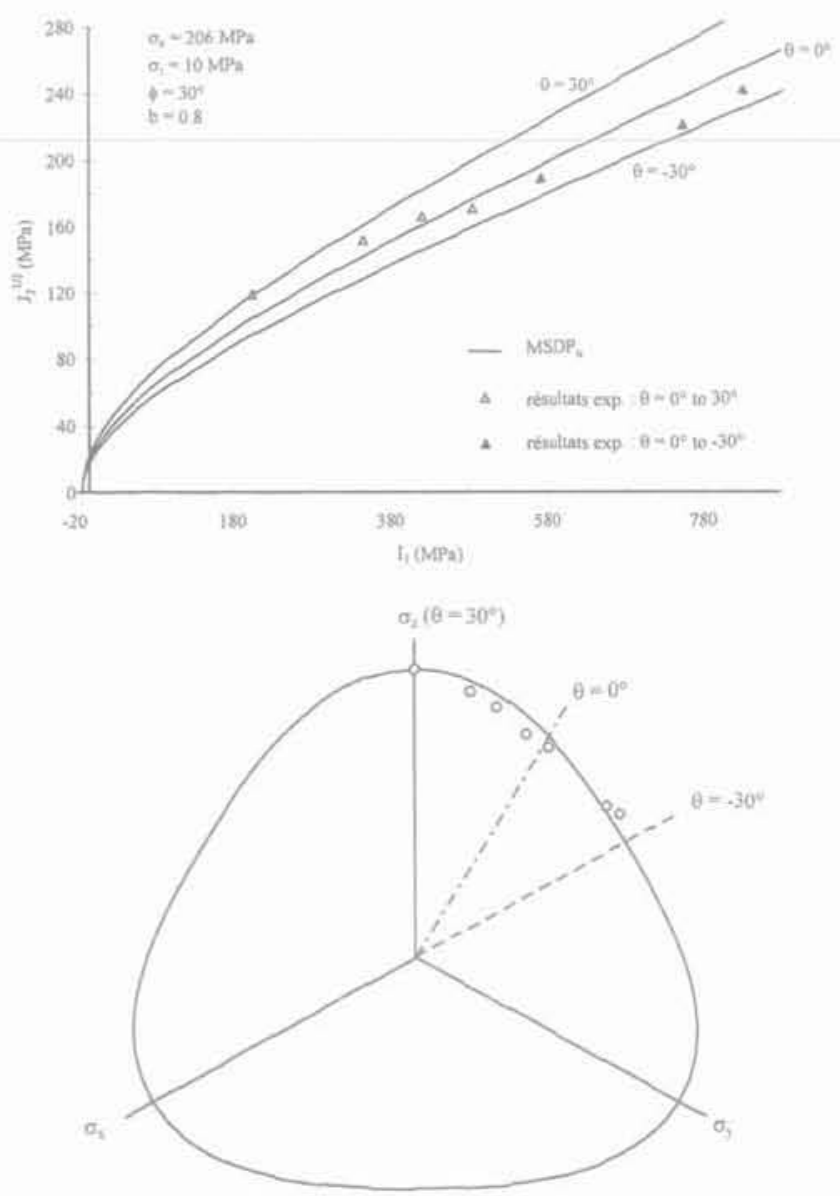

FiG.3 Le critère MSDP $_{u}$ appliqué à la rupture d'un calcaire sous différentes conditions de chargement (données tirées de Hoskins. 1969) : (a) dans le plan $\sqrt{J_{2}}-I_{1}$; (b) dans le plan $\pi$.

The MSDP criterion applied to limestone submitted to different loading conditions (data taken from Hoskins, 1969) : (a) in the $\sqrt{J_{2}}-I_{4}$ plane; (b) in the $\pi$ plane.

$\left(\sigma_{4}\right)$ est considérée comme une contrainte principale, parallèle à l'axe du forage. Elle peut être mesurée directement ou estimée à partir du poids de terre.

Pour l'analyse de la condition de rupture, on utilise la notion de facteur de sécurité (FS) défini comme suit : 
$\mathrm{FS}=\frac{\mathrm{F}_{0} \mathrm{~F}}{\sqrt{\mathrm{J}_{2}}}=$

Capacité (contrainte admissible calculée par MSDP $\mathrm{u}_{\mathrm{u}}$

Demande (contrainte déviatorique induite)

Une rupture aura théoriquement lieu lorsque la valeur du facteur de sécurité est de un. Dans un forage, la zone d'écaillage s'étend d'abord aux points B' et B (cf. Fig. 4) et elle s'arrête au point C où FS a aussi une valeur unitaire.

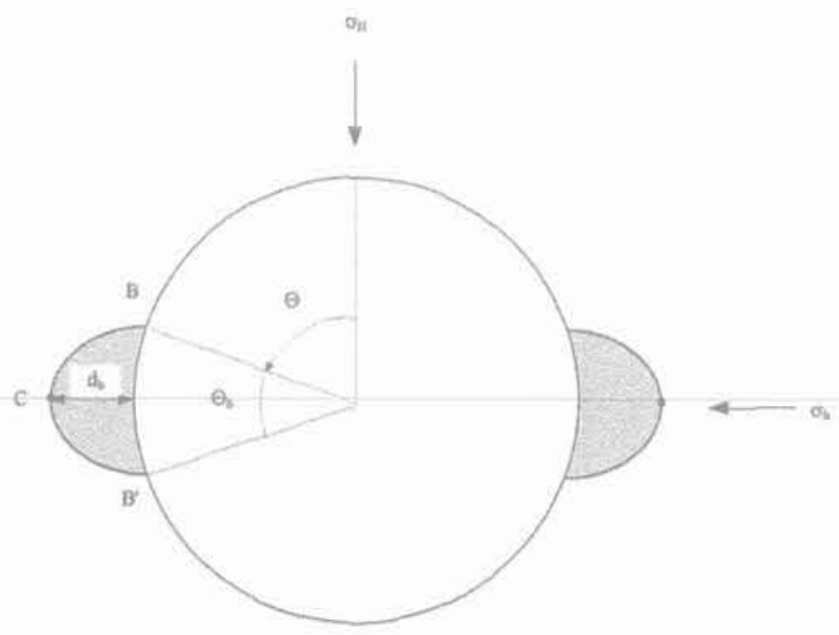

F1G.4 Définition des caractéristiques de la zone d'écaillage autour d'un trou de forage. Definition of a borehole breakout characteristics.

Pour une valeur de $\sigma_{v}$ donnée, l'angle critique $\Theta_{b}$ et la profondeur $\mathrm{d}_{b}$ de la zone d'écaillage peuvent être utilisés pour estimer les valeurs des contraintes principales horizontales majeure et mineure $\left(\sigma_{\mathrm{H}}\right.$ et $\left.\sigma_{h}\right)$. Considérant que l'angle de l'écaillage $\Theta_{b}$ reste inchangé une fois que l'écaillage s'amorce pour un état de contrainte donné (Zheng et al., 1989), les contraintes critiques aux points B et B' peuvent être calculées à l'aide de solutions numériques ou de la solution analytique proposée par Hiramatsu et Oka (1962). Cette dernière peut s'exprimer de la façon suivante :

$$
\begin{aligned}
& \sigma_{1}=\sigma_{H}+\sigma_{h}-2\left(\sigma_{H}-\sigma_{h}\right) \cos 2 \Theta \\
& \sigma_{2}=\sigma_{v}-2 v\left(\sigma_{H}-\sigma_{h}\right) \cos 2 \Theta \\
& \sigma_{3}=0
\end{aligned}
$$

où les contraintes principales majeure, intermédiaire et mineure $\left(\sigma_{1}, \sigma_{3}\right.$ et $\left.\sigma_{3}\right)$ correspondent aux contraintes tangentielle, axiale et radiale autour du trou respectivement; $v$ est le coefficient de Poisson; $\Theta$ est l'angle de la zone d'écaillage entre le point B (ou B') et la direction de la contrainte principale horizontale maximale $\sigma_{H}(\Theta$ $\left.=90^{\circ}-\Theta_{b} / 2\right)$. L'angle de Lode correspondant est donné par:

$$
\theta=\tan ^{-1} \frac{\sigma_{1}-2 \sigma_{2}}{\sqrt{3} \sigma_{1}}
$$

et les invariants des contraintes induites sont donnés par :

$$
\begin{gathered}
\mathrm{L}_{1}=\sigma_{1}+\sigma_{2} \\
\sqrt{J_{2}}=\frac{1}{\sqrt{3}}\left(\sigma_{1}^{2}-\sigma_{1} \sigma_{2}+\sigma_{2}^{2}\right)^{1 / 2}
\end{gathered}
$$

En utilisant l'équation 6 avec FS $=1$, on obtient une équation qui décrit le rapport entre les contraintes horizontales maximale et minimale $\sigma_{\mathrm{H}}$ et $\sigma_{\mathrm{h}}$ et l'angle de la zone d'écaillage $\Theta_{b}$.

$$
\sqrt{J_{2}}\left[\sigma_{H}, \sigma_{h}, \Theta\right]_{B}=F_{0}\left[\sigma_{H}, \sigma_{h}, \Theta\right]_{B} F_{n}\left[\sigma_{H}, \sigma_{h}, \Theta\right]_{B}
$$

On peut aussi établir une autre équation pour l'état critique au point $C$ :

$$
\sqrt{J_{2}}\left[\sigma_{\mathrm{H}}, \sigma_{\mathrm{h}}, \mathrm{d}_{\mathrm{b}}\right]_{\mathrm{C}}=\mathrm{F}_{\mathrm{o}}\left[\sigma_{\mathrm{H}}, \sigma_{\mathrm{h}}, \mathrm{d}_{\mathrm{b}}\right]_{\mathrm{C}} \mathrm{F}_{n}\left[\sigma_{\mathrm{H}}, \sigma_{\mathrm{h}}, \mathrm{d}_{\mathrm{b}}\right]_{\mathrm{C}}
$$

La résolution des équations (11) et (12), où il n'y a que deux inconnues, permet de calculer les contraintes principales horizontales maximale et minimale $\sigma_{H}$ et $\sigma_{\mathrm{h}}$. Toutefois, puisqu'il n'existe pas de solution analytique appropriée pour évaluer l'état de contrainte critique au point C (équation 12), une méthode numérique (telles les méthodes des éléments finis ou des éléments frontières) doit être utilisée.

\section{4}

\section{Application de la méthode}

\section{1}

\section{Présentation générale}

Pour illustrer l'application de cette procédure, on utilise d'abord, de façon schématisée, une situation représentative d'un forage. Le trou a un rayon de $2,5 \mathrm{~cm}$ et se trouve dans une roche élastique linéaire, homogène et isotrope. Les constantes élastiques de la roche sont fixées arbitrairement à $E=50 \mathrm{GPa}$ et $\mathrm{v}=0,25$. Les courbes d'enveloppe de résistance de la roche, décrites par $\mathrm{MSDP}_{\mathrm{u}}$, sont montrées à la figure 5. Pour cet exemple illustratif, les contraintes en place seraient de $\sigma_{\mathrm{H}}=300 \mathrm{MPa}, \sigma_{\mathrm{h}}=100 \mathrm{MPa}$, et $\sigma_{\mathrm{v}}=125 \mathrm{MPa}$.

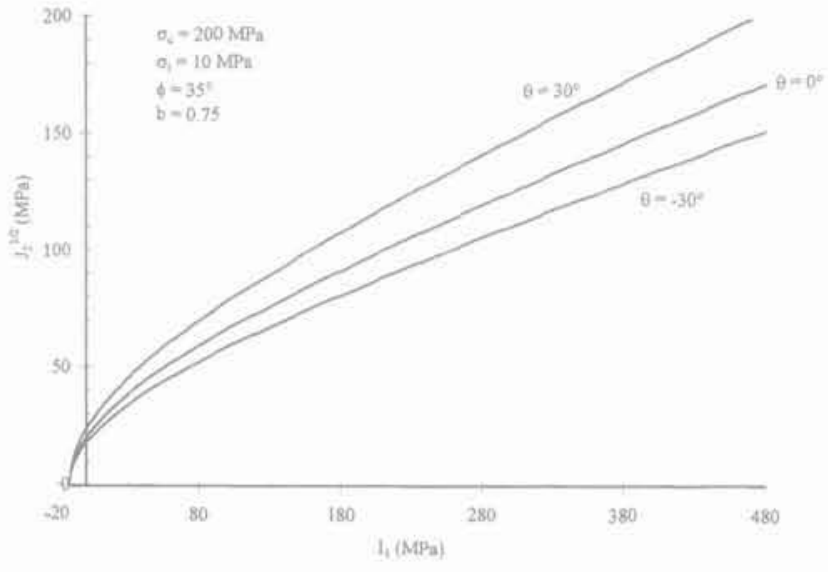

FIG.5 Surface de rupture définie avec MSDP pour le problème analysé.

Failure surface defined by MSDP for the sample problem investigated.

La figure 6 montre la représentation du développement progressif de la zone de rupture en utilisant le critère MSDP et un calcul fait à l'aide d'un code tridimensionnel basé sur la méthode des éléments frontières, développé par Li (1997). L'angle $\Theta$ où apparaît la rupture, qui constitue une donnée du problème 


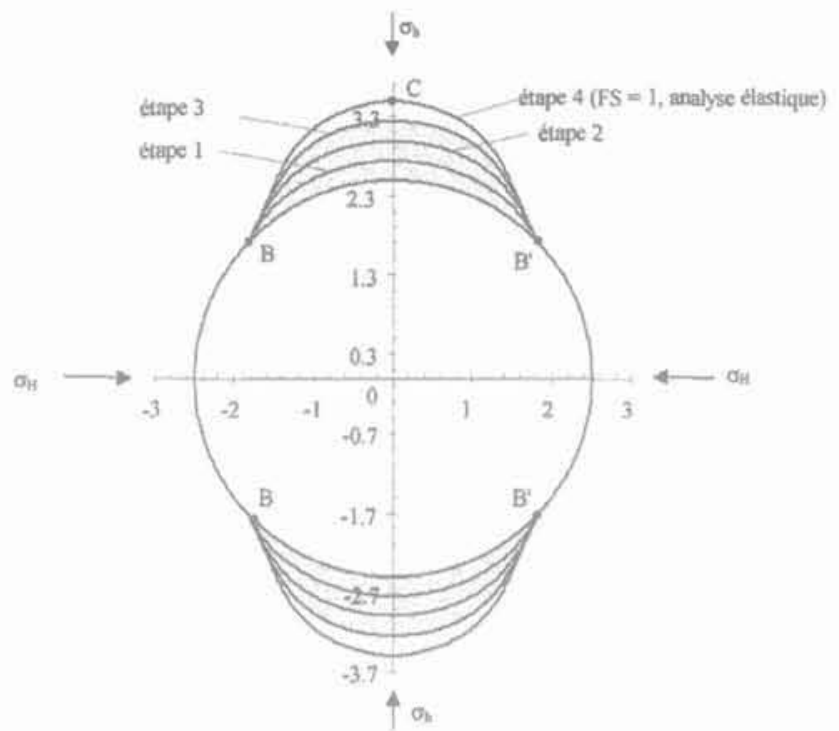

FIG. 6 Modélisation de la propagation de la zone de rupture autour du trou de forage; les zones ombragées sont enlevées pour l'étape suivante de calcul.

Modeling of breakout extension - shaded areas are removed for the next calculation step.

(observée dans le trou), est de $43^{\circ}\left(\Theta_{\mathrm{b}}=94^{\circ}\right)$. Même si la méthode de discontinuité de déplacement constante (une méthode par éléments frontières) ne permet pas de calculer très précisément l'état des contraintes directement sur les frontières (e.g. Crouch, 1976; Li, 1997), elle permet néanmoins d'évaluer, tel que montré à la figure 6 , la zone de progression de la rupture proche de la paroi du trou.

L'extension complète de la zone d'écaillage $d_{b}$ ne peut pas être obtenue uniquement à partir d'analyses élastiques linéaires. Par conséquent, on doit réaliser des analyses en définissant les propriétés post-pic pour les zones amenées à la rupture. Compte tenu de la nature fragile du comportement des roches (avec une résistance post-pic très faible pour une petite valeur de $\sigma_{3}$ ), les matériaux dans les zones de rupture sont simplement enlevés pour ensuite procéder à une nouvelle étape de calcul. Une fois que l'on a positionné le contour final de rupture correspondant à la dernière analyse élastique avec FS $=1$ au point $C$ (position connue par observation), on peut estimer l'état des contraintes à ce point connaissant la géométrie de la zone d'écaillage (angle $\Theta_{b}$ et distance $\mathrm{d}_{\mathrm{b}}$ ). Une telle approximation permet d'évaluer assez précisément les conditions qui prévalent le long de l'interface où se développe la rupture.

En utilisant le critère MSDP , on peut tracer une carte des contours du facteur de sécurité (FS) au point $C$ dans le plan $\sigma_{\mathrm{H}}-\sigma_{\mathrm{h}}$ selon les équations (6) et (12) (Fig. 7). De façon similaire, la solution d'Hiramatsu et Oka (1962) (ou une solution numérique) combinée au critère MSDP permettent de tracer d'autres contours de FS dans le même plan $\sigma_{\mathrm{H}}-\sigma_{\mathrm{h}}$ pour les points $B$ et $B^{\prime}$ (aussi montrés sur la figure 7 pour l'exemple analysé ici). La superposition de ces deux séries de contours sur la carte de FS permet de déduire directement les valeurs de $\sigma_{\mathrm{H}}$ et de $\sigma_{h}$. Dans le présent exemple, on trouverait :

$$
\sigma_{\mathrm{H}}=302 \mathrm{MPa} \text { et } \sigma_{\mathrm{h}}=101 \mathrm{MPa}
$$

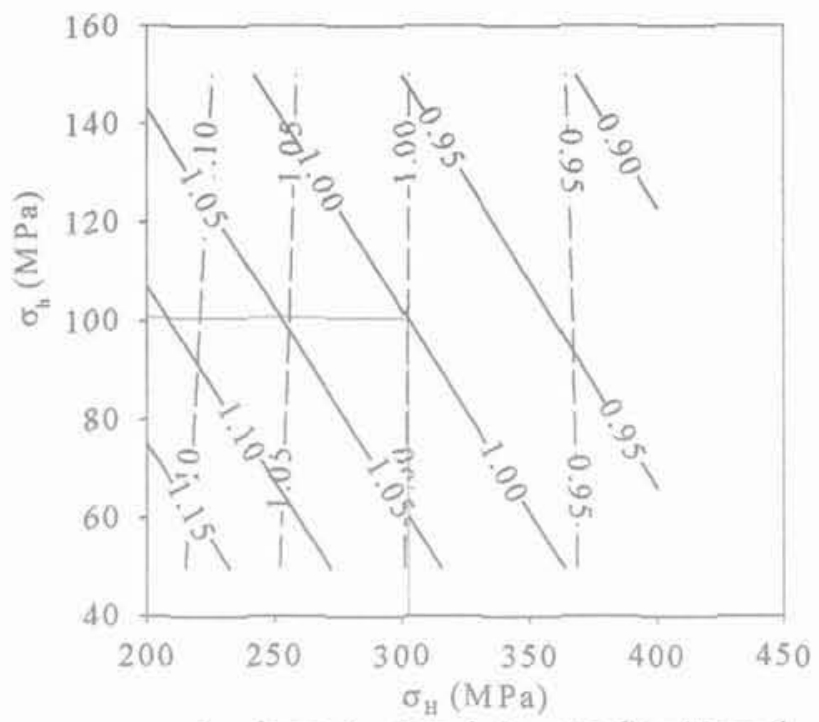

_- selon la profondeur de la zone d'écaillage d

- selon l'angle de la zone d'écaillage $\Theta_{b}$

FiG.7 Carte des isocontours de facteurs de sécurité dans le plan $\sigma_{h}-\sigma_{\mu}$, qui varient selon l'angle et la profondeur de la zone d'écaillage.

Isocontour map of safety factors in the $\sigma_{\mathrm{n}}-\sigma_{h}$ plane, which vary according to the angle and depth of the breakout zone.

pour l'état critique FS = 1, ce qui représenterait une faible différence par rapport aux valeurs des contraintes imposées. Évidemment, il ne s'agit que d'un cas fictif, où les conditions imposées correspondent aux conditions calculées. Cela permet néanmoins d'illustrer comment la méthode peut être appliquée.

Malheureusement, il ne semble pas exister de cas documentés où l'ensemble de l'information requise pour appliquer cette méthode serait disponible. On retrouve par contre divers cas documentés dans la littérature où une partie de l'information est fournie. C'est à partir de tels cas que l'on applique directement la méthode dans ce qui suit.

\section{$4 ?$}

\section{Application spécifique}

Afin d'illustrer, à partir d'un cas réel, l'application de la procédure proposée, on utilise les données de Song et Haimson (1997). Dans ce cas-ci, on suppose que $\sigma_{v}$ et $\sigma_{h}$ sont connues et l'on cherche à déterminer $\sigma_{H}$ pour $\mathrm{FS}=1$. L'équation (11) se réduit donc à :

$$
\sqrt{J_{2}}\left[\sigma_{H}, \Theta\right]_{B}=F_{0}\left[\sigma_{H}, \Theta\right]_{B} F_{\pi}\left[\sigma_{H}, \Theta\right]_{B}
$$

où $\Theta\left(\right.$ ou $\Theta_{\text {b }}$ ) est une valeur connue suite à l'observation de la zone d'écaillage.

L'équation (13) ne contient qu'une seule inconnue, soit $\sigma_{H}$, qui peut être obtenue par la résolution de l'équation. Celle-ci ne peut toutefois être résolue que d'une manière itérative. Pour solutionner celle-ci, une approche graphique est proposée.

Une plage plausible des valeurs de $\sigma_{H}$ est d'abord établie (de $\sigma_{\text {Hryn }}$ à $\sigma_{\text {Hrax }}$ ) basée sur une connaissance des valeurs regionales (contraintes naturelles) ou locales (contraintes induites). La valeur de $\sigma_{H}$ affecte l'angle de Lode, si bien qu'à chaque valeur de $\sigma_{\mathrm{H}}$ cor- 
respond une courbe de rupture différente dans le plan $\sqrt{J_{2}}-I_{1}$. La figure 8 a montre une représentation schématisée du critère MSDP $\mathrm{M}_{\mathrm{u}}$ pour les angles de Lode correspondant à $\sigma_{H \text { min }}$ et $\sigma_{\text {Hmax' }}$ avec les valeurs calculées correspondantes de $\sqrt{J_{2}}, I_{1}\left(\sigma_{\text {Himin }}\right)$ et de $\sqrt{J_{2}}$, $\mathrm{I}_{1}\left(\sigma_{\text {Himax }}\right)$. A partir de la droite qui lie ces deux points (A et $B$ ), deux nouveaux points d'intersection ( $F$ et $G$ ) sont obtenus sur les courbes de rupture appropriées ; on peut alors déduire les valeurs de $\sqrt{J_{2}}, I_{1}\left(\sigma_{H 1}\right)$ et de $\sqrt{J_{2}}$, $\mathrm{L}_{1}\left(\sigma_{\mathrm{H} 2}\right)$. Les valeurs de $\sigma_{\mathrm{H} 1}$ et de $\sigma_{\mathrm{H} 2}$ peuvent ensuite être calculées à partir de l'équation (9), chacune correspondant à un angle de Lode qui peut être établi selon les équations (7) et (8) (soit $\theta\left(\sigma_{H_{1}}\right)$ et $\theta\left(\sigma_{H_{2}}\right)$ ). L'angle de Lode $\theta$ associé à la valeur réelle de $\sigma_{H}$ peut subséquemment être obtenu par interpolation graphique (Fig. 8b), ou à partir de l'équation suivante :

$$
\frac{\theta\left(\sigma_{H}\right)-\theta\left(\sigma_{H \min }\right)}{\theta\left(\sigma_{\text {Hmax }}\right)-\theta\left(\sigma_{H \min }\right)}=\frac{\theta\left(\sigma_{H}\right)-\theta\left(\sigma_{H 1}\right)}{\theta\left(\sigma_{H 2}\right)-\theta\left(\sigma_{H 1}\right)}
$$
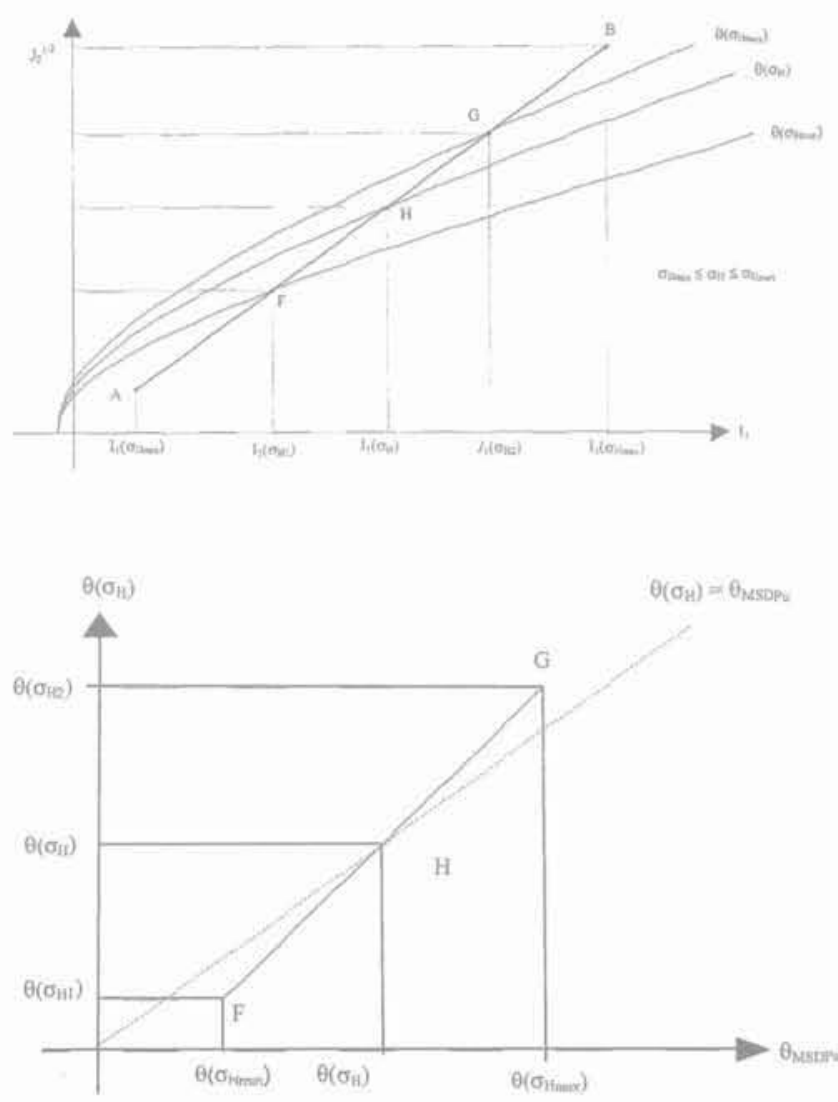

Approche graphique pour déduire la valeur de $\sigma_{\mathrm{H}}$ : (a) MSDP dans le plan $\sqrt{J_{2}}-h_{1}$ avec divers angles de Lode: (b) interpolation pour trouver le point $\mathrm{H}$ où $\theta\left(s_{\mathrm{H}}\right)$ est donné à partir des équations fournies dans le texte.

Graphical method to deduce the value of $\sigma_{\mathrm{H}}$ : (a) the MSDP $\mathrm{P}_{u}$ criterion in the $\sqrt{\mathrm{J}_{2}}-I_{1}$ plane with different Lode angles; (b)

interpolation to find point $\mathrm{H}$, where $\theta\left(\sigma_{H}\right)$ is obtained from equations given in the text.
Une troisième courbe de $\mathrm{MSDP}_{\mathrm{u}}$, avec $\theta\left(\sigma_{\mathrm{H}}\right)$, peut être tracée dans le plan $\sqrt{J_{2}}-I_{1}$ (Fig. 8a). On obtient alors directement le point d'intersection correspondant à $I_{1}\left(\sigma_{H}\right)$. La valeur de $\sigma_{H}$ est finalement déduite selon les équations (7) et (9). La figure $8 \mathrm{~b}$ illustre schématiquement comment converger au point $\mathrm{H}$ et vers la valeur réelle de $\sigma_{H}$ en utilisant les valeurs de $\theta$ déduites de l'équation (9) et de l'application du critère MSDP $_{\mathrm{u}}$ avec les équations (7), (8) et (13).

Cette procédure graphique est aussi schématisée au tableau 1 . Elle est appliquée aux résultats d'essais de laboratoire publiés par Song et Haimson (1997) qui ont réalisé des essais sur des blocs de grès de Berea et de granite de Westerly, percés de trous. Les valeurs déduites de la procédure sont montrées au tableau II. On constate une bonne correspondance entre les valeurs calculées et la valeur de la contrainte imposée pour les deux types de roches (tableau II).

\section{4 \\ Discussion}

La procédure présentée ici permet d'estimer les contraintes perpendiculaires à l'axe d'un trou de forage, si cet axe correspond à l'orientation d'une des contraintes principales. Cette technique s'applique d'abord à l'estimation des contraintes naturelles, mais elle pourrait aussi s'appliquer aux cas où l'on souhaite évaluer ou confirmer les valeurs des contraintes induites autour des excavations. Dans ce dernier cas, l'observation en trou de forage constitue un moyen simple et économique de confirmer les résultats de calculs sur l'état des contraintes générées autour des excavations, une information particulièrement utile lorsque l'on s'intéresse à l'évaluation de la stabilité (e.g. Simon et al., 1998).

La méthode proposée est relativement simple et facile à appliquer lorsque les informations requises sont disponibles. Pour son application, il faut toutefoís conserver certains points spécifiques à l'esprit. Ainsi la taille $\mathrm{d}_{\mathrm{b}}$ de la zone d'écaillage autour d'un trou de forage ne peut pas être déterminée uniquement par un modèle élastique linéaire, puisque la distribution de contraintes change avec la progression de la zone de rupture (Cheatham, 1993). Comme la résistance postpic des roches fragiles est usuellement très faible, on peut, en première approximation, négliger celle-ci en éliminant les matériaux dans les zones de rupture, tel que montré ici.

La méthode d'estimation des contraintes présentée dans cet article, basée sur le critère MSDP, est en principe indépendante du comportement de la roche. On pourrait, dans les cas où cela s'avère nécessaire, appliquer un modèle constitutif plus élaboré permettant de prendre en compte, par exemple, la non-linéarité, les effets du temps et de l'échelle, et les caractéristiques du massif rocheux (e.g. Aubertin et al., 1999b), et ce tout en suivant la même procédure pour estimer les contraintes en place.

\section{5}

\section{Conclusion}

Dans cet article, les auteurs présentent une méthode pour déduire l'ampleur des contraintes horizontales 
TABLEAU1 Synthèse de la procédure pour déduire la contrainte principale maximale horizontale $\sigma_{\mathrm{H}}$. Schematic presentation of the procedure for the evaluation of the maximum horizontal principal stress $\sigma_{\mathrm{H}}$.

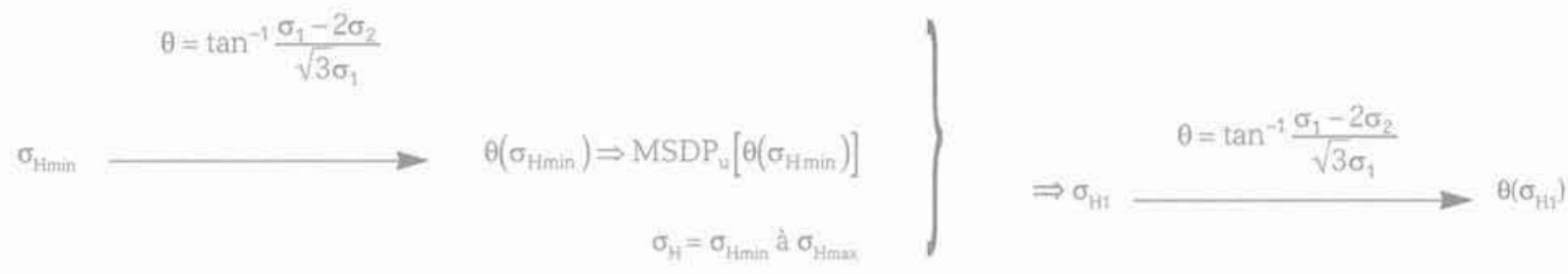

$$
\begin{aligned}
& \theta=\tan ^{-1} \frac{\sigma_{1}-2 \sigma_{2}}{\sqrt{3 \sigma_{1}}} \\
& \sigma_{\text {Hinax }} \longrightarrow \theta\left(\sigma_{\operatorname{Hax}}\right) \Rightarrow \operatorname{MSDP}_{u}\left[\theta\left(\sigma_{\mathrm{H} \max }\right)\right] \\
& \sigma_{\mathrm{H}}=\sigma_{\mathrm{H} \min } \text { à } \sigma_{\mathrm{Hmax}}
\end{aligned}
$$

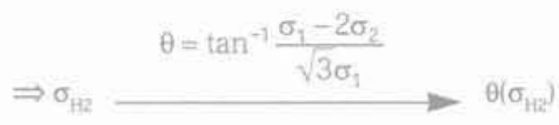

$\frac{\theta\left(\sigma_{H}\right)-\theta\left(\sigma_{H \min }\right)}{\theta\left(\sigma_{H \max }\right)-\theta\left(\sigma_{\mathrm{Hmin}}\right)}=\frac{\theta\left(\sigma_{H}\right)-\theta\left(\sigma_{H 1}\right)}{\theta\left(\sigma_{H 2}\right)-\theta\left(\sigma_{H 1}\right)} \Rightarrow \theta\left(\sigma_{H}\right) \Rightarrow I_{1}\left(\sigma_{H}\right) \Rightarrow \sigma_{H}$

TABLEAUIII Comparaison entre les contraintes imposées et les valeurs calculées par inversion (résultats

\begin{tabular}{|c|c|c|c|c|c|c|}
\hline \multirow[t]{2}{*}{ Poches } & \multicolumn{3}{|c|}{ Contraintes imposées (MPa) } & \multirow{2}{*}{$\begin{array}{c}\text { Angles d'écaillage } \\
\text { observés } \Theta_{\text {l }}\end{array}$} & \multirow{2}{*}{$\begin{array}{l}\text { Valeurs calculées } \\
\qquad \sigma_{\mathrm{H}}(\mathrm{MPa})\end{array}$} & \multirow[t]{2}{*}{ Difference $(\%)$} \\
\hline & $\sigma_{\mathrm{h}}$ & $\sigma_{x}$ & $\sigma_{i 1}$ & & & \\
\hline Grès de Berea & $\begin{array}{l}20 \\
30\end{array}$ & $\begin{array}{l}30 \\
40\end{array}$ & $\begin{array}{l}80 \\
90\end{array}$ & $\begin{array}{l}31^{\circ} \\
35^{\circ}\end{array}$ & $\begin{array}{l}75 \\
80\end{array}$ & $\begin{array}{r}6,3 \\
11,1\end{array}$ \\
\hline Granite de Westerly & $\begin{array}{l}30 \\
30\end{array}$ & $\begin{array}{l}40 \\
40\end{array}$ & $\begin{array}{l}160 \\
180\end{array}$ & $\begin{array}{l}14^{\circ} \\
33^{\circ}\end{array}$ & $\begin{array}{l}154 \\
164\end{array}$ & $\begin{array}{l}3,8 \\
8,9\end{array}$ \\
\hline
\end{tabular}
expérimentaux tírés de Song et Haimson, 1997).

Comparaison between the back-calculated values and the imposed stresses (experimental resuits taken from Song and Haimson, 1997).

maximale et minimale à partir de la géométrie de la zone d'écaillage autour d'un trou de forage, en appliquant le critère de rupture multiaxial MSDP, L'application de cette méthode est d'abord montrée à l'aide d'un exemple illustratif. Comme cas particulier, la contrainte horizontale principale maximale $\sigma_{\mathrm{H}}$ a été cal- culée de façon inverse en utilisant des résultats d'essais de laboratoire tirés de la littérature. La méthode, simple et économique, peut être utilisée pour estimer l'ampleur de deux des trois contraintes principales naturelles ou des contraintes induites autour des excavations.

\section{REMERCIEMENTS}

Une partie de ce travail a été financée par des subventions du NSERC (OGP 0089749) et de IIRSST. En particulier, le premier auteur souhaite remercier ITRSST pour sa bourse de recherche post-doctorale. Nous remercions aussi le Professeur B. Haimson qui a fourni des informations supplémentaires concernant les résultats d'essais de laboratoire. 
Aglawe J.P. - Unstable and violent failure around underground openings in highly stresses ground. These de doctorat, Queen's University at Kingston, 1999.

Anderson R. Moos D. Goldberg D. Zoback M.D. - Hydraulically induced breakouts : a new method for determining stress orientation in the crust? EOS, vol. $66, \mathrm{n}^{\circ} 18,1985$, p. 363 .

Aubertin M., Simon R. - A multiaxial failure criterion that combines two quadric surfaces. Tools and Techniques in Rock Mechanics, M. Aubertin. F. Hassan. H. Mitri, eds. Balkerna, Rotterdam, 1996, p. $1729-1736$.

Aubertin M., Simon R. - Un critère de rupture multiaxial pour matẻriaux fragiles Revue canadienne de Génie civil, vol. 25 $n^{\circ}$ 2, 1998, p. 277-290.

Aubertin M., Li L., Simon R., Khalfi S. Formulation and application of a short term strength criterion for isotopic rocks. Canadian Geotechnical Journal (accepté pour publication), 1999a.

Aubertin M., Li L., Simon R., Khalfi S. - A unified representation of the damage and failure criteria for rocks and rock masses. Proc. 9th Congr. ISRM, Paris, August, 1999 G. Vouille, P. Berest eds. Balkema, 1999 b, p. 843-848.

Barton C.A., Zoback M.D., Burns K.L. - In situ stress orientation and magnitude at the Fenton geothermal site, New Mexico, determined from wellbore breakouts. Geophysical Research Let ters, vol. $15, n^{\circ} 5,1988$, p. $467-470$.

Bell J.S., Gough D.1. - Northeast-southwest compressive stress in Alberta : evidence from oil wells. Earth and Planetary Science Letters, vol, $45, n^{\circ} 2,1979$, p. $475-482$.

Cheatham J.B. Jr, - A new hypothesis of explain stability of borehole breakouts. International Journal of Rock Mechanics and Mining Sciences \& Geomechanics Abstracts, vol. $30, n^{\circ} 7,1993$, p. 195-1101.

Crouch S.L. - Solution of plane elasticity problems by the discontinuity method. International Journal of Numerica Methods in Engineering, vol. 10, 1976 p. $301-343$

Elata D. - Modeling wellbore breakouts. International Journal of Rock Mechanics and Mining Sciences, vol. $34, n^{\circ} 3-4$ 1997, paper $n^{\circ}$ 072, CD-POM. Elsevier.

Guenot A. - Borehole breakouts and stress fields. International Journal of Rock Mechanics and Mining Sciences, vol. 26, $n^{\circ}$ 3/4, 1989, p. 185-195.

Hamson B.C., Herrick C.G. - In situ stress evaluation from borehole breakouts. In: Research \& Enqineering Applications in Rock Masses ; Proc. 26th US Symp, Rock Mech., June 26-28, 1985, Rapid City, SD, Ashworth E, ed. Rotterdam : Balkema, 1985, vol. 2, p. 1207-1218.
Haimson B.C., Song I. - Laboratory study of borehole breakouts in Cordova Cream : a case of shear failure mechanism. International Journal of Rock Mechanics and Mining Sciences, vol. 30 , $n^{\circ}$ 7. 1993, p. 1047-1056.

Hickman S.H., Healy J.H., Zoback M.D. In situ stress, natural fracture distribution, and borehole elongation in the Auburn Geothermal well, Auburn. New York. Journal of Geophysical Research. vol. $90, n^{\circ}$ B7, 1985, p. $5497-5512$.

Hiramatsu Y., Oka Y. - Stress around a shaft or level excavated in ground with a three-dimensional stress state. Memoirs of the Faculty of Engineering, Kyoto University, vol. 24, 1962, p. 56-76

Hoskins E.R. - The failure of thick-wallned hollow cylinders of isotropic rock. International Journal of Rock Mechanics And Mining Sciences, vol. 6, 1969, p. 99-125.

Lee M. Haimson B. - Laboratory study of breakouts in Lac du Bonnet granite : a case of extensile failure mechanism. International Journal of Rock Mechanics and Mining Sciences \& Geornechanics Abstracts, vol. $30, n^{\circ} 7,1993$, p. 10391945.

Leeman E.R. - The measurement of stress in rock. Journal of South African Institute of Mining and Metallurgy, 1964, p. 45114.

Li L. - Étude expérimentale du comportement hydromécanique d'une fracture. Thèse de doctorat, Institut de Physique du Globe de Paris, Université Paris 7. Denis-Diderot, 1997

Li L., Aubertin M. Simon R. - Stability analyses of underground openings using a multiaxial failure criterion. GeoEnqineering for Undercround Facilities Proceedings, 3rd National Conference of the Geo-Institute of ASCE, June 13-17. 1999. Champaingn-Urbana. Fernande: G., Bauer R.A., eds., 1999, p. 471-482.

Martin C.D. - The strength of massive lac du Bonnet granite around underground openings. Thèse de doctorat, University of Manitoba, 1993

Mastin L.G. - Development of borehole breakouts in sandstone. Mémoire de Maîtrise, Sanford University, Palo Alto, 1984

Mastin L.G. - Effect of borehole deviation on breakout orientations. Journal of Geophysical Research, vol. 93. $\mathrm{n}^{\circ} \mathrm{B} 8$, 1988, p. 9187-9195

Maury V - Observations, researches and recent results about failure mechanisms around single galleries. Proceedings, 6th International Congress on Rock Mechanics, Herget G., Vongpaisal S,, eds, vol. 2, 1987, p. 1119-1128.

Peska P., Zoback M.D. - Compressive and tensile failure of inclined well bore and determination of in situ stress and rock strength. Journal of Geophysical Research, vol. $100, n^{\circ}$ B7, 1995 , p. 12781 12811.

Plumb R.A. - Fracture patterns associated with incipient wellbore breakouts. Rock at Great Depth, V. Maury, D. Fourmaintraux, eds. Balkema, Rotterdam, vol. 2. 1989, p. 761-768.

Plumb R., Hickman S.H. - Stress-induced borehole elongation: a comparison between the four-arm dipmeter and the borehole televiewer in the Auburn geothermal well. Journal of Geophysical Research, vol. $90, \pi^{\circ}$ B7, 1985, p. 5513 5521.

Qian W., Pedersen L.B. - Inversion of berehole breakout orientation data, Journa of Geophysical Research, vol. 96, n ${ }^{\circ}$ B12. 1991, p. 20093-20107.

Schwartz A.E. - Failure of rock in the triaxial test. Proceedings, 6th U.S. Symposium Rock Mechanics, 1964, p. 109151.

Simon R., Aubertin M., Auer L., Gill D.E, Mitri H.S. - A study of destress blasting effect on the rockburst potential of an underground mine in hard rocks. In Proc, 3rd North American Rock Mech. Symp., Cancun, Mexico, 1998, vol. 2, p. $285-294$

Song L. Haimson B.C. - Polyaxial strength criteria and their use in estimating in situ stress magnitudes from borehole breakout dimensions. International Journal of Rock Mechanics \& Mining Science, vol, 34, $\mathrm{n}^{\mathrm{se}} 3-4,1997$, paper $\mathrm{n}^{\circ}$ 116, CD-ROM, Elsevier.

Vernik L., Zoback M.D. - Estimation of maximum horizontal principal stress magnitude from stress-induced well bore breakouts in the Cajon Pass scientific research borehole. Journal of Geophysical Research, vol. 97, n B4. 1992, p. 5109-5112.

Yin J.M., Cornet F.H, - Integrated stress determination by joint inversion of hydraulic tests and focal mechanisms. Geophysical Research Letters, vol, 21, $n^{\circ} 24,1994$, p. 2645-2648.

Zheng Z, Cook N.G.W, - Generation and analysis of stable wellbore cross section. EOS, vol. 66, n 46, 1985, p. 1056.

Zheng Z, Kemeny J., Cook N.G.W. - Analysis of borehole breakouts. Journal of Geophysical Research, vol. 94, n ${ }^{\circ}$ B6. 1989, p. $7171-7182$

Zoback M.D., Moos D., Mastin L., Anderson R.N. - Well bore breakouts and in situ stress. Journal of Geophysical Research. vol. $90, n^{\circ}$ BT, 1685 , p. $5523-5530$.

Zoback M.L. - First- and second-order patterns of stress in the lithospheric stress patterns, Journal of Geophysical Research, vol. 97, n B8, 1992, p. 11703 11728. 